\title{
TRPC3: A New Target for Therapeutic Strategies in Chronic Pain-DAG-mediated Activation of Non-selective Cation Currents and Chronic Pain
}

(Mol Pain 2014;10:43)

\author{
Meng Xia, ${ }^{1,2}$ Dan Liu, ${ }^{3}$ and Chun Yao ${ }^{1 *}$ \\ ${ }^{1}$ Institute of Foundational Research of Chinese Medicine, College of Basic Medicine, Guangxi University of Chinese Medicine, Guangxi, China; \\ ${ }^{2}$ Institute of Foundational Research of Chinese Medicine, Hubei University for Nationalities, Huiei, China; and ${ }^{3}$ Department of Design and \\ Technology, Tianjin Wuqing Hospital of Chinese Medicine, Tianjin, China
}

\section{Summary}

Chronic visceral pain is a typical condition associated with inflammation. The mechanism of visceral pain in chronic abdominal pain syndromes, such as irritable bowel syndrome and chronic pancreatitis, is poorly understood and its treatment has been largely empirical, with an outcome that is variable and frequently unsatisfactory. Human and experimental studies have indicated a critical role of neuronal mechanisms that result in peripheral and central sensitization. ${ }^{1-3}$ The nociceptive sensitization, literally meaning sensitization to noxious stimuli, begins with the primary afferent nociceptor, a nerve whose cell body lies in the dorsal root ganglia (DRG). DRG is located next to the spinal cord and has 2 branches: a long peripheral one terminating in the target tissue and a shorter central process that ends in the dorsal horn of the spinal cord. The peripheral process of these neurons senses tissue injury via a variety of receptors that respond to specific physical or chemical factors in the injured environment. In studying visceral pain, several visceral nociceptors have been identified as significant factors in inflammatory condition. For instance, A-type potassium channel is downregulated in chronic pancreatitis. $^{2}$ Others, including transient receptor potential vanilloid $1,{ }^{4}$ nerve growth factor, ${ }^{5}$ the protease activated receptor 2 (PAR2), ${ }^{6}$ and transforming growth factor beta $1,{ }^{7}$ are upregulated. In a further mechanism study on those nociceptors in thoracic/ lumbar DRG, a prolonged intracellular calcium elevation is commonly associated with the neuronal hyper-excitability or hypersensitivity, contributing to nociception and chronic pain (hyperalgesia or allodynia).

There are 2 distinct mechanisms proposed in modulating the intracellular calcium level when noxious stimuli activating the cognate membrane receptors at the surface of nociceptors. ${ }^{8}$ Store-operated calcium entry (SOCE) involves in activation of calcium channels by inositol 1,4,5-trisphosphate which is the key for calcium depletion from endoplasmic reticulum calcium stores;

Received: May 11, 2015 Revised: June 20, 2015 Accepted: June 24, 2015

(c) This is an Open Access article distributed under the terms of the Creative Commons Attribution Non-Commercial License (http://creativecommons. org/licenses/by-nc/4.0) which permits unrestricted non-commercial use, distribution, and reproduction in any medium, provided the original work is properly cited.

*Correspondence: Chun Yao, MD Institute of Foundational Research of Chinese Medicine, College of Basic Medicine, Guangxi University of Chinese Medicine, 179 Mingxiu Dong Rd., Nanning, Guangxi, China

Tel: +86-13877171987, Fax: +86-771-3137517, E-mail: gxtcm2015@139.com

Financial support: This work was supported by the Natural Science Foundation of China grants (81302930).

Conflicts of interest: None.

Author contributions: Meng Xia wrote the draft; Dan Liu organized and mediated the present study; and Chun Yao have made revisions and corrections on the manuscript. 
receptor-operated calcium entry (ROCE) involves in activation of calcium permeable channels directly by DAG. ${ }^{9,10}$ It remains unknown whether ROCE mechanism sensitizes the sensory neuron, however, SOCE component participates in calcium homeostasis and plays a significant role in chronic pain pathologies.

Recently, Alkhani et al ${ }^{11}$ published "Contribution of TRPC3 to store-operated calcium entry and inflammatory transductions in primary nociceptors" in Molecular Pain. This study reveals a major contribution of transient receptor potential cation channels (TRPC) to neuronal calcium homeostasis in somatosensory pathways. These channels are non-selectively permeable to cations, with a selectivity of calcium over sodium variable among the different members. Here, TRPC engages in both SOCE and ROCE in control of calcium influx that triggers calcium-dependent pathways and peripheral sensitization. Therefore TRPC is functionally coupled to several inflammatory transductions, including UTP/P2Y2 and proteases/PAR2 signaling complexes. Logically, this unique dual contribution to SOCE and ROCE defines the calcium-permeable TRPC3 channel as a key regulator of calcium homeostasis in DRG neurons in normal or pathological pain conditions. Therefore, TRPC3 is a new target for therapeutic strategies in chronic pain.

\section{Comments}

Prolonged intracellular calcium elevation contributes to sensitization of nociceptors and chronic pain in inflammatory conditions. Many strategies have been applied in control of visceral pain in current treatment or in clinical trials by focusing on a blockage of noxious stimuli. For instance, the US Food and Drug Administration has voted unanimously that antibodies against nerve growth factor should re-enter clinical trials for pain. ${ }^{12}$ These strategies are mainly based on the foreigner noxious agent or environment, such as released chemicals, or "inflammatory soup". It is well known that the noxious agent is always increased at the site of the chronic inflammatory pain; it is just beginning to know that the intracellularly endogenous agent such as DAG is also over produced in inflammatory pain. From Alkhani et al, ${ }^{11}$ it is more evident that DAG is a real trigger to prolong intracellular calcium elevation contributing to sensitization of nociceptors and chronic pain in inflammatory conditions.

There are the following evidences to support their claims: first, TRPC 3 is physically expressed in small and medium diameter sensory neurons that are for responsible to pain, this is consistent with an earlier study reported by Kress et al. ${ }^{13}$ Second, these neurons evoked a significant increase of $\mathrm{Gd}^{3+}$-sensitive SOCE conductance in following thapsigargin-induced calcium store depletion. With further activation by DAG (an analog of OAG), the increased $\mathrm{Ca}^{2+}$ influx can be potently inhibited by SKF96365, suggesting this channel in SOCE response is activated through phospholipase $\mathrm{C}$ pathway DAG-gated. Third, to check the role of TRPC3 in SOCE response in DRG neurons, Alkhani et al. applied RNA interference with a subunit specific to GFP-tagged TRPC3 shRNA, its knockdown efficacy was confirmed by Pyr10, a selective TRPC3 antagonist, indicating a significant role of TRPC3 in SOCE. Furthermore, activation of $\mathrm{P} 2 \mathrm{Y} 2$ purinoceptors (P2Y2 receptor) or PAR2 triggered a strong increase in intracellular calcium which is attributed from TRPC3 overexpression; on other hand, it was suppressed in knockdown of native TRPC 3 and suppressed by Pyr10. Authors in this study and other studies ${ }^{13}$ have also found that TRPC1 is present in DRG sensory neuron, including small and medium sized neurons. TRPC1 function has also been linked to ubiquitous stromal interaction molecule 1 (STIM1)-dependent SOCE. It is likely that TRPC 1 and 3 both contribute to SOCE in the current study.

Nevertheless, these data show a robust link between TRPC3 channel activation and pro-inflammatory receptors through DAG overexpression. DAG mediates a calcium influx by operating both of SOCE and ROCE. It is reasonable to infer that TRPC 3 can couple SOCE/ROCE and DAG as pro-inflammatory metabotropic receptor in peripheral sensitization. Therefore, TRPC 3 could be a potential target for therapeutic strategies in chronic pain conditions.

\section{References}

1. Knowles $\mathrm{CH}$, Aziz Q. Basic and clinical aspects of gastrointestinal pain. Pain 2009;141:191-209.

2. Pasricha PJ. Unraveling the mystery of pain in chronic pancreatitis. Nat Rev Gastroenterol Hepatol 2012;9:140-151.

3. Sikandar S, Dickenson AH. Visceral pain: the ins and outs, the ups and downs. Curr Opin Support Palliat Care 2012;6:17-26.

4. Zhu Y, Colak T, Shenoy M, et al. Nerve growth factor modulates TRPV1 expression and function and mediates pain in chronic pancreatitis. Gastroenterology 2011;141:370-377.

5. Winston J, Toma H, Shenoy M, Pasricha PJ. Nerve growth factor regulates VR-1 mRNA levels in cultures of adult dorsal root ganglion neurons. Pain 2001;89:181-186.

6. Bueno L. Protease activated receptor 2: a new target for IBS treatment. Eur Rev Med Pharmacol Sci 2008;12(suppl 1):95-102.

7. Zhu Y, Colak T, Shenoy M, et al. Transforming growth factor beta induces sensory neuronal hyperexcitability, pancreatic pain and hy- 
peralgesia in rats with chronic pancreatitis. Mol Pain 2012;8:65.

8. Ammann, RW, Akovbiantz A, Largiader F, Schueler G. Course and outcome of chronic pancreatitis. Longitudinal study of a mixed medical-surgical series of 245 patients. Gastroenterology 1984;86(5 Pt 1): 820-828.

9. Ossovskaya VS, Bunnett NW. Protease-activated receptors: contribution to physiology and disease. Physiol Rev 2004;84:579-621.

10. Trejo J. Protease-activated receptors: new concepts in regulation of $G$ protein-coupled receptor signaling and trafficking. J Pharmacol Exp Ther 2003;307:437-442.

11. Alkhani H, Ase AR, Grant R, O'Donnell D, Groschner K, Séguéla P. Contribution of TRPC 3 to store-operated calcium entry and in- flammatory transductions in primary nociceptors. Mol Pain 2014; $10: 43$

12. Talkington M. FDA advisory panel gives the green light to restart NGF antibody trials. Pain Research Forum - Progress through collaboration. Available URL: http://www.painresearchforum.org/ news/14439-fda-gives-green-light-restart-ngf-antibody-trials (accessed 23 Jun 2015).

13. Kress M. Karasek J, Ferrer-Montiel AV, Scherbakov N, Haberberger RV. TRPC channels and diacylglycerol dependent calcium signaling in rat sensory neurons. Histochem Cell Biol 2008;130: 655-667. 